\begin{tabular}{|c|l|}
\hline Title & Ground State Energy of the Polaron in the Relativistic Quantum Electrodynamics \\
\hline Author(s) & Sasaki, Itaru \\
\hline Citation & Hokkaido University Preprint Series in Mathematics, 737, 1-8 \\
\hline Issue Date & 2005 \\
\hline DOI & 10.14943/83887 \\
\hline Doc URL & http://hdl.handle.net/2115/69545 \\
\hline Type & bulletin (article) \\
\hline File Information & pre737.pdf \\
\hline
\end{tabular}

Instructions for use 


\title{
Ground State Energy of the Polaron in the Relativistic Quantum Electrodynamics
}

\author{
Itaru Sasaki \\ Department of Mathematics, \\ Hokkaido University, \\ Sapporo 060-0810, Japan \\ e-mail: i-sasaki@math.sci.hokudai.ac.jp
}

July 30, 2005

\begin{abstract}
We consider the polaron model in the relativistic quantum electrodynamics(QED). We prove that the ground state energy of the model is finite for all values of the fine-structure constant and the ultraviolet cutoff $\Lambda$. Moreover we give an upper bound and a lower bound of the ground state energy.

Key words: relativistic QED; ground state energy, polaron model.
\end{abstract}

\section{Introduction and Main Results}

We consider the relativistic quantum electrodynamics(QED) for a fixed total momentum - the polaron model of the relativistic QED. The Hamiltonian, which describes a Dirac particle minimally coupled to the quantized radiation field, commutes with the total momentum operator and has a direct integral decomposition with respect to the total momentum operator([1], [2]). Each fibre in this direct integral decomposition is just the Hamiltonian of the polaron we consider. The Hilbert space of the polaron model is defined by

$$
\mathcal{F}:=\mathbb{C}^{4} \otimes \mathcal{F}_{\mathrm{b}}\left(L^{2}\left(\mathbb{R}^{3} \times\{1,2\}\right)\right),
$$


where

$$
\mathcal{F}_{\mathrm{b}}\left(L^{2}\left(\mathbb{R}^{3} \times\{1,2\}\right)\right):=\bigoplus_{n=0}^{\infty}\left[\bigotimes_{s}^{n}\left(L^{2}\left(\mathbb{R}^{3} \times\{1,2\}\right)\right)\right]
$$

is the photon Fock space $\left(\otimes_{s}^{n}\right.$ denotes $n$-fold symmetric tensor product). For a closable operator $T$ on $L^{2}\left(\mathbb{R}^{3} \times\{1,2\}\right)$ we denote by $\mathrm{d} \Gamma_{\mathrm{b}}(T)$, the second quantization operator of $T$ (see [4]). Let $a(f), f \in L^{2}\left(\mathbb{R}^{3} \times\{1,2\}\right)$ be the annihilation operator on the photon Fock space. For a function $g_{j} \in L^{2}\left(\mathbb{R}^{3} \times\{1,2\}\right), j=1,2,3$, we set

$$
A_{j}:=a\left(g_{j}\right)+a\left(g_{j}\right)^{*}, \quad j=1,2,3 .
$$

Let $\left\{\alpha_{1}, \alpha_{2}, \alpha_{3}, \beta\right\}$ be the $4 \times 4$-Dirac matrices, i.e., $\left\{\alpha_{i}, \alpha_{j}\right\}=2 \delta_{i, j},\left\{\alpha_{i}, \beta\right\}=0, \beta^{2}=$ $1, i, j=1,2,3$. Here $\{A, B\}:=A B+B A$. For three objects $a_{1}, a_{2}, a_{3}$ we set $\mathbf{a}=$ $\left(a_{1}, a_{2}, a_{3}\right)$, and write $\mathbf{a} \cdot \mathbf{b}:=\sum_{j=1}^{3} a_{j} b_{j}$, provided that $a_{j} b_{j}$ and $\sum_{j=1}^{3} a_{j} b_{j}$ are defined.

The Hamiltonian of the polaron model we consider is

$$
H(\mathbf{p}):=\boldsymbol{\alpha} \cdot \mathbf{p}+M \beta+\mathrm{d} \Gamma_{\mathrm{b}}(\omega)-\boldsymbol{\alpha} \cdot \mathrm{d} \Gamma_{\mathrm{b}}(\mathbf{k})-q \boldsymbol{\alpha} \cdot \mathbf{A},
$$

where $\mathbf{p} \in \mathbb{R}^{3}$ is the fixed total momentum, $M \geq 0$ is the mass of the Dirac particle, $q \in \mathbb{R}$ is a constant proportional to the fine-structure constant, and $\omega=|\mathbf{k}|$ is the 1-photon Hamiltonian $\left(\mathbf{k} \in \mathbb{R}^{3}\right)$. Note that we omit the symbol $\otimes$ between the Hilbert space for the Dirac matrices $\mathbb{C}^{4}$ and the photon Fock space $\mathcal{F}_{\mathrm{b}}\left(L^{2}\left(\mathbb{R}^{3} \times\{1,2\}\right)\right)$. The most important example of $\left\{g_{j}\right\}_{j=1}^{3}$ is of the form

$$
f_{j}(\mathbf{k}, r):=\frac{\chi_{\Lambda}(\mathbf{k})}{|\mathbf{k}|^{1 / 2}} e_{j}^{(r)}(\mathbf{k})
$$

where the measurable functions $\mathbf{e}^{(1)}(\mathbf{k}), \mathbf{e}^{(2)}(\mathbf{k})$ are the polarization vectors:

$$
\mathbf{k} \cdot \mathbf{e}^{(r)}(\mathbf{k})=0, \quad \mathbf{e}^{(r)}(\mathbf{k}) \cdot \mathbf{e}^{(s)}(\mathbf{k})=\delta_{r, s}, \quad \text { a.e. } \mathbf{k} \in \mathbb{R}^{3}, \quad r, s=1,2,
$$

and $\chi_{\Lambda}(\mathbf{k})$ is the characteristic function of the ball $\left\{\mathbf{k} \in \mathbb{R}^{3}|| \mathbf{k} \mid<\Lambda\right\}, \Lambda>0$.

We define

$$
E_{0}(\mathbf{p}):=\inf _{\substack{\Psi \in \operatorname{Dom}(H(\mathbf{p})) \\\|\Psi\|=1}}\langle\Psi, H(\mathbf{p}) \Psi\rangle
$$

the ground state energy of $H(\mathbf{p})$, where "Dom" means operator domain. We assume the following:

Hypothesis I. $g_{j} \in \operatorname{Dom}\left(\omega^{-1 / 2}\right) \cap \operatorname{Dom}(\omega),\left\langle g_{j}, g_{\ell}\right\rangle \in \mathbb{R}, j, \ell=1,2,3$. 
It should be noted that it is highly non-trivial whether or not $E_{0}(\mathbf{p})$ is finite, because $H(\mathbf{p})$ contains the term $-\boldsymbol{\alpha} \cdot \mathrm{d} \Gamma_{\mathrm{b}}(\mathbf{k})$. This is the main problem discussed in the present paper.

We prove that the ground state energy $E_{0}(\mathbf{p})$ is finite under suitable conditions:

Theorem 1.1. Assume Hypothesis I, and

$$
G(\mathbf{g}):=\inf _{\mathbf{k} \in \mathbb{R}^{3} \backslash\{0\}} \frac{1}{|\mathbf{k}|} \sum_{r=1,2} \int_{\mathbb{R}^{3}} \frac{\left|\mathbf{k} \cdot \mathbf{g}\left(\mathbf{k}^{\prime}, r\right)\right|^{2}}{|\mathbf{k}|\left|\mathbf{k}^{\prime}\right|-\mathbf{k} \cdot \mathbf{k}^{\prime}} \mathrm{d} \mathbf{k}^{\prime}<\infty .
$$

Then, the ground state energy $E_{0}(\mathbf{p})$ is finite:

$$
E_{0}(\mathbf{p})>-\infty
$$

In particular, if $g_{j}=f_{j}, j=1,2,3$, the ground state energy $E_{0}(\mathbf{p})$ is finite.

For a vector $u \in \mathbb{C}^{4}$, we set $a_{j}:=\left\langle u, \alpha_{j} u\right\rangle_{\mathbb{C}^{4}}$, and

$$
\mathcal{E}(\Lambda, u):=\mathbf{p} \cdot \mathbf{a}+M\langle u, \beta u\rangle+4 \pi \Lambda q^{2} \frac{1-|\mathbf{a}|^{2}}{|\mathbf{a}|} \log \left(\frac{1+|\mathbf{a}|}{1-|\mathbf{a}|}\right)-4 \pi \Lambda q^{2} .
$$

In the physical case (i.e. the function $g_{j}$ 's are given by (5)), the lower bound of $E_{0}(\mathbf{p})+\sqrt{|\mathbf{p}|^{2}+M^{2}}$ are proportional to $\Lambda$ :

Theorem 1.2. Let $g_{j}=f_{j}, j=1,2,3$. Then

$$
\begin{aligned}
& C_{1} \Lambda-\sqrt{|\mathbf{p}|^{2}+M^{2}} \leq E_{0}(\mathbf{p}), \\
& E_{0}(\mathbf{p}) \leq C_{2}(\Lambda)
\end{aligned}
$$

where

$$
\begin{aligned}
C_{1} & :=\inf _{\epsilon, \epsilon^{\prime}>0}\left\{\epsilon|q|+16 \pi q^{2}+\left(\epsilon^{\prime}+\frac{1}{\epsilon^{\prime}}\right) 4 \pi q^{2}, \sqrt{\frac{4 \pi|q|}{3 \epsilon}+\left(1+\frac{1}{\epsilon^{\prime}}\right) 4 \pi q^{2}}\right\}, \\
C_{2}(\Lambda) & :=\inf _{\substack{u \in \mathbb{C}^{4} \\
\|u\|_{\mathbb{C}^{4}=1}}} \mathcal{E}(\Lambda, u) .
\end{aligned}
$$

\section{Proof of Theorem 1.1 and 1.2}

Lemma 2.1. Let $A$ be a positive self-adjoint operator on a Hilbert space $\mathcal{H}$. Let $B$ be a symmetric operator with $\operatorname{Dom}(A) \subset \operatorname{Dom}(B)$ and

$$
\|B \Psi\| \leq\|A \Psi\|, \quad \Psi \in \operatorname{Dom}(A) .
$$

Then, for all $\Psi \in D(A),\langle\Psi,(A+B) \Psi\rangle \geq 0$. 
Proof. By the Kato-Rellich theorem([4]), for all $\epsilon \in(-1,1), A+\epsilon B$ is self-adjoint and $A+\epsilon B \geq 0$. Therefore $\langle\Psi,(A+B) \Psi\rangle \geq 0$ for all $\Psi \in \operatorname{Dom}(A)$.

By this lemma, it suffices to show that there exists a constant $E \geq 0$ such that

$$
\left\|\left(\mathrm{d} \Gamma_{\mathrm{b}}(\omega)+E\right) \Psi\right\|^{2} \geq\left\|\boldsymbol{\alpha} \cdot\left(\mathrm{d} \Gamma_{\mathrm{b}}(\mathbf{k})+q \mathbf{A}\right) \Psi\right\|^{2}, \quad \Psi \in \operatorname{Dom}\left(\mathrm{d} \Gamma_{\mathrm{b}}(\omega)\right) .
$$

We use the following representation for $\boldsymbol{\alpha}$-matrices:

$$
\alpha_{j}=\left[\begin{array}{cc}
\sigma_{j} & 0 \\
0 & -\sigma_{j}
\end{array}\right], \quad j=1,2,3
$$

with $\left(\sigma_{1}, \sigma_{2}, \sigma_{3}\right)$ being the Pauli matrices. Using anticommutation relation of $\alpha_{1}, \alpha_{2}$ and $\alpha_{3}$, we have

$\left\|\boldsymbol{\alpha} \cdot\left(\mathrm{d} \Gamma_{\mathrm{b}}(\mathbf{k})+q \mathbf{A}\right) \Psi\right\|^{2}=\sum_{j=1}^{3}\left\|\left(\mathrm{~d} \Gamma_{\mathrm{b}}\left(k_{j}\right)+q A_{j}\right) \Psi\right\|^{2}-q\left\langle\Psi, \mathbf{S} \cdot\left[a(i \mathbf{k} \times \mathbf{g})+a(i \mathbf{k} \times \mathbf{g})^{*}\right] \Psi\right\rangle$,

where $S_{j}=\sigma_{j} \oplus \sigma_{j}, j=1,2,3$. The Hilbert space $\mathbb{C}^{4} \otimes\left[\otimes_{s}^{n} L^{2}\left(\mathbb{R}^{3} \times\{1,2\}\right)\right]$ is naturally embedded in $L^{2}\left(\mathbb{R}^{3} \times\{1,2\} ; \mathbb{C}^{4} \otimes\left[\otimes_{s}^{(n-1)} L^{2}\left(\mathbb{R}^{3} \times\{1,2\}\right)\right]\right)$. For a vector $\Psi \in \mathbb{C}^{4} \otimes$ $\left[\otimes_{s}^{n} L^{2}\left(\mathbb{R}^{3} \times\{1,2\}\right)\right]$, we denote its value at point $(\mathbf{k}, r) \in \mathbb{R}^{3} \times\{1,2\}$ by $\Psi(\mathbf{k}, r, \cdot)$.

For $\Psi=\left(\Psi^{(n)}\right)_{n=0}^{\infty} \in \mathcal{F}$, we define

$$
a^{(r)}(\mathbf{k}) \Psi:=\left(\Psi^{(1)}(\mathbf{k}, r), \sqrt{2} \Psi^{(2)}(\mathbf{k}, r, \cdot), \cdots \sqrt{n} \Psi^{(n)}(\mathbf{k}, r, \cdot), \cdots\right) \in \mathcal{F}, \quad \mathbf{k} \in \mathbb{R}^{3}, r=1,2,
$$

a Fock space valued function. This operator $a^{(r)}(\mathbf{k})$ is the distributional kernel of the annihilation operator.

Lemma 2.2. For all $\Psi \in \operatorname{Dom}\left(\mathrm{d} \Gamma_{\mathrm{b}}(\omega)\right)$ and $\epsilon>0$, the following inequality holds:

$$
\left|q\left\langle\Psi, \mathbf{S} \cdot\left[a(i \mathbf{k} \times \mathbf{g})+a(i \mathbf{k} \times \mathbf{g})^{*}\right] \Psi\right\rangle\right| \leq|q| \epsilon\left\langle\Psi, \mathrm{d} \Gamma_{\mathrm{b}}(\omega) \Psi\right\rangle+\frac{|q|}{\epsilon}\langle\mathbf{g}, \omega \mathbf{g}\rangle\|\Psi\|^{2},
$$

where $\langle\mathbf{g}, \omega \mathbf{g}\rangle:=\sum_{j=1}^{3}\left\langle g_{j}, \omega g_{j}\right\rangle$. 
Proof.

$$
\begin{aligned}
\text { l.h.s of }(18) & =2|q|\left|\operatorname{Re} \oint_{\mathbb{R}^{3}}\left\langle\Psi,-i \mathbf{S} \cdot(\mathbf{k} \times \mathbf{g}(\mathbf{k}, r)) a^{(r)}(\mathbf{k}) \Psi\right\rangle \mathrm{d} \mathbf{k}\right| \\
& \leq 2|q| \oiint_{\mathbb{R}^{3}}\|\mathbf{S} \cdot(\mathbf{k} \times \mathbf{g}(\mathbf{k}, r)) \Psi\|\left\|a^{(r)}(\mathbf{k}) \Psi\right\| \mathrm{d} \mathbf{k} \\
& =2|q| \oint_{\mathbb{R}^{3}}|\mathbf{k}|^{1 / 2}|\mathbf{g}(\mathbf{k}, r)| \cdot\left\||\mathbf{k}|^{1 / 2} a^{(r)}(\mathbf{k}) \Psi\right\| \cdot\|\Psi\| \mathrm{d} \mathbf{k} \\
& \leq 2|q|\langle\mathbf{g}, \omega \mathbf{g}\rangle^{1 / 2}\left[\oiint_{\mathbb{R}^{3}}\left\||\mathbf{k}|^{1 / 2} a^{(r)}(\mathbf{k}) \Psi\right\|^{2}\right]^{1 / 2}\|\Psi\| \\
& \leq|q| \epsilon\left\langle\Psi, \mathrm{d} \Gamma_{\mathrm{b}}(\omega) \Psi\right\rangle+\frac{|q|}{\epsilon}\langle\mathbf{g}, \omega \mathbf{g}\rangle\|\Psi\|^{2},
\end{aligned}
$$

where $f:=\sum_{r=1,2} \int$.

Lemma 2.3. For all $\Psi \in \operatorname{Dom}\left(\mathrm{d} \Gamma_{\mathrm{b}}(\omega)\right)$ and $\epsilon>0$, the following inequality holds:

$$
\left\langle\Psi, \mathbf{A}^{2} \Psi\right\rangle \leq\left(2+\epsilon+\frac{1}{\epsilon}\right)\left\langle\omega^{-1 / 2} \mathbf{g}, \omega^{-1 / 2} \mathbf{g}\right\rangle\left\langle\Psi, \mathrm{d} \Gamma_{\mathrm{b}}(\omega) \Psi\right\rangle+\left(1+\frac{1}{\epsilon}\right)\langle\mathbf{g}, \mathbf{g}\rangle\|\Psi\|^{2}
$$

Proof.

$$
\begin{aligned}
\left\langle\Psi, \mathbf{A}^{2} \Psi\right\rangle \leq & \sum_{j=1}^{3}\left[(1+\epsilon)\left\|a\left(g_{j}\right) \Psi\right\|^{2}+\left(1+\frac{1}{\epsilon}\right)\left\|a\left(g_{j}\right)^{*} \Psi\right\|^{2}\right] \\
\leq & \sum_{j=1}^{3}\left[(1+\epsilon)\left\||\mathbf{k}|^{-1 / 2} g_{j}\right\|^{2} \cdot\left\|\mathrm{d} \Gamma_{\mathrm{b}}(\omega)^{1 / 2} \Psi\right\|^{2}\right. \\
& \left.+\left(1+\frac{1}{\epsilon}\right)\left\||\mathbf{k}|^{-1 / 2} g_{j}\right\|^{2} \cdot\left\|\mathrm{d} \Gamma_{\mathrm{b}}(\omega)^{1 / 2} \Psi\right\|^{2}+\left(1+\frac{1}{\epsilon}\right)\left\|g_{j}\right\|^{2} \cdot\|\Psi\|^{2}\right] .
\end{aligned}
$$

The following Lemma is the most important fact in the proof of Theorem 1.1:

Lemma 2.4. For all $\Psi \in \operatorname{Dom}\left(\mathrm{d} \Gamma_{\mathrm{b}}(\omega)\right)$, the following inequality holds:

$$
\begin{aligned}
& \left\|\mathrm{d} \Gamma_{\mathrm{b}}(\omega) \Psi\right\|^{2}-\sum_{j=1}^{3}\left\|\mathrm{~d} \Gamma_{\mathrm{b}}\left(k_{j}\right) \Psi\right\|^{2}-q\left\langle\mathrm{~d} \Gamma_{\mathrm{b}}(\mathbf{k}) \Psi, \mathbf{A} \Psi\right\rangle-q\left\langle\mathbf{A} \Psi, \mathrm{d} \Gamma_{\mathrm{b}}(\mathbf{k}) \Psi\right\rangle \\
& \geq-4 q^{2} G(\mathbf{g})\left\langle\Psi, \mathrm{d} \Gamma_{\mathrm{b}}(\omega) \Psi\right\rangle-q\left\langle\Psi,\left(a(\mathbf{k} \cdot \mathbf{g})^{*}+a(\mathbf{k} \cdot \mathbf{g})\right) \Psi\right\rangle .
\end{aligned}
$$

Proof. We define

$$
F:=\frac{\mathbf{k} \cdot \mathbf{g}\left(\mathbf{k}^{\prime}, \mu\right)}{|\mathbf{k}| \cdot|\mathbf{k}|-\mathbf{k} \cdot \mathbf{k}^{\prime}}
$$


For all $\Psi \in \operatorname{Dom}\left(\mathrm{d} \Gamma_{\mathrm{b}}(\omega)\right)$, we have

$$
\begin{aligned}
\text { l.h.s of }(20)= & \mathcal{F}_{\mathbb{R}^{3}} \mathrm{~d} \mathbf{k} \mathcal{F}_{\mathbb{R}^{3}}{ }^{\prime} \mathrm{d} \mathbf{k}^{\prime}\left(|\mathbf{k}| \cdot\left|\mathbf{k}^{\prime}\right|-\mathbf{k} \cdot \mathbf{k}^{\prime}\right)\|(b-2 q F) a \Psi\|^{2} \\
& -q\left\langle\Psi,\left[a(\mathbf{k} \cdot \mathbf{g})+a(\mathbf{k} \cdot \mathbf{g})^{*}\right] \Psi\right\rangle \\
& -4 q^{2} \mathcal{f}_{\mathbb{R}^{3}} \mathrm{~d} \mathbf{k}\left[\mathscr{f}_{\mathbb{R}^{3}}^{\prime} \mathrm{d} \mathbf{k}^{\prime}\left(|\mathbf{k}| \cdot\left|\mathbf{k}^{\prime}\right|-\mathbf{k} \cdot \mathbf{k}^{\prime}\right)^{-1}\left|\mathbf{k} \cdot \mathbf{g}\left(\mathbf{k}^{\prime}, \mu\right)\right|^{2}\right]\|a \Psi\|^{2},
\end{aligned}
$$

where $a:=a^{(r)}(\mathbf{k}), b:=a^{(\mu)}\left(\mathbf{k}^{\prime}\right)$, and $\mathfrak{f}^{\prime}:=\sum_{\mu=1,2} \int$. Since $|\mathbf{k}| \cdot\left|\mathbf{k}^{\prime}\right|-\mathbf{k} \cdot \mathbf{k}^{\prime} \geq 0$, the inequality (20) holds.

Proof of Theorem 1.1. Using Lemmas 2.2 - 2.4, we get

$$
\begin{aligned}
\left.\left(\mathrm{d} \Gamma_{\mathrm{b}}(\omega)+E\right)^{2}-\sum_{j=1}^{3}\left(\mathrm{~d} \Gamma_{\mathrm{b}}\left(k_{j}\right)+q A_{j}\right)^{2}-q \mathbf{S} \cdot\left[a(i \mathbf{k} \times \mathbf{g})+a(i \mathbf{k} \times \mathbf{g})^{*}\right)\right] \\
\geq 2 E \mathrm{~d} \Gamma_{\mathrm{b}}(\omega)+E^{2}-4 q^{2} G(\mathbf{g}) \mathrm{d} \Gamma_{\mathrm{b}}(\omega)-q\left[a(\mathbf{k} \cdot \mathbf{g})^{*}+a(\mathbf{k} \cdot \mathbf{g})\right]-|q| \mathrm{d} \Gamma(\omega) \\
\quad-|q|\langle\mathbf{g}, \omega \mathbf{g}\rangle-4\left\langle\omega^{-1 / 2} \mathbf{g}, \omega^{-1 / 2} \mathbf{g}\right\rangle \mathrm{d} \Gamma_{\mathrm{b}}(\omega)-2\langle\mathbf{g}, \mathbf{g}\rangle,
\end{aligned}
$$

in the sense of quadratic form on $\operatorname{Dom}\left(\mathrm{d} \Gamma_{\mathrm{b}}(\omega)\right)$. Since $a(\mathbf{k} \cdot \mathbf{g})+a(\mathbf{k} \cdot \mathbf{g})^{*}$ is $\mathrm{d} \Gamma_{\mathrm{b}}(\omega)^{1 / 2}$ bounded, for a large $E>0$ we have

$$
\left.\left(\mathrm{d} \Gamma_{\mathrm{b}}(\omega)+E\right)^{2}-\sum_{j=1}^{3}\left(\mathrm{~d} \Gamma_{\mathrm{b}}\left(k_{j}\right)+q A_{j}\right)^{2}-q \mathbf{S} \cdot[a(i \mathbf{k} \times \mathbf{g})+a(i \mathbf{k} \times \mathbf{g}))\right] \geq 0 .
$$

By Lemma 2.1, for a large $E \geq 0$, we obtain

$$
\mathrm{d} \Gamma_{\mathrm{b}}(\omega)-\boldsymbol{\alpha} \cdot \mathrm{d} \Gamma_{\mathrm{b}}(\mathbf{k})-q \boldsymbol{\alpha} \cdot \mathbf{A} \geq-E
$$

in the sense of quadratic form on $\operatorname{Dom}\left(\mathrm{d} \Gamma_{\mathrm{b}}(\omega)\right)$. This inequality implies that $E_{0}(\mathbf{p})$ is finite.

Next we show that $G(\mathbf{f})<\infty$ if $g_{j}=f_{j}(j=1,2,3)$. By the definitions of $\mathbf{e}^{(r)}(\mathbf{k})$, the vectors $\mathbf{k} /|\mathbf{k}|, \mathbf{e}^{(1)}(\mathbf{k}), \mathbf{e}^{(2)}(\mathbf{k})$ are the orthonormal basis of $\mathbb{C}^{3}$. Therefore

$$
G(\mathbf{f})=\sup _{\mathbf{k} \in \mathbb{R}^{3} \backslash\{0\}} \frac{1}{|\mathbf{k}|} \int_{\mathbb{R}^{3}} \frac{\chi_{\Lambda}\left(\mathbf{k}^{\prime}\right) \mathrm{d} \mathbf{k}^{\prime}}{|\mathbf{k}|\left|\mathbf{k}^{\prime}\right|-\mathbf{k} \cdot \mathbf{k}^{\prime}} \cdot \frac{1}{\left|\mathbf{k}^{\prime}\right|}\left[|\mathbf{k}|^{2}-\frac{\left(\mathbf{k} \cdot \mathbf{k}^{\prime}\right)^{2}}{\left|\mathbf{k}^{\prime}\right|^{2}}\right]=\int_{\mathbb{R}^{3}} \frac{\chi_{\Lambda}\left(\mathbf{k}^{\prime}\right)}{\left|\mathbf{k}^{\prime}\right|^{2}} \mathrm{~d} \mathbf{k}^{\prime}=4 \pi \Lambda .
$$

Proof of Theorem 1.2. First we show (10). We set $g_{j}=f_{j}(j=1,2,3)$. It is easy to see that

$$
H(\mathbf{p}) \geq-\sqrt{|\mathbf{p}|^{2}+M^{2}}+\mathrm{d} \Gamma_{\mathrm{b}}(\omega)-\boldsymbol{\alpha} \cdot \mathrm{d} \boldsymbol{\Gamma}_{\mathrm{b}}(\mathbf{k})-q \boldsymbol{\alpha} \cdot \mathbf{A}
$$


By the definition of $\mathbf{e}^{(r)}(\mathbf{k})$, we have $\mathbf{k} \cdot \mathbf{f}(\mathbf{k}, r)=0\left(\mathbf{k} \in \mathbb{R}^{3}, r=1,2\right)$. Therefore, using Lemmas $2.2-2.4$, we have

$$
\begin{aligned}
& \left(\mathrm{d} \Gamma_{\mathrm{b}}(\omega)+C_{1} \Lambda\right)^{2}-\left(\mathrm{d} \Gamma_{\mathrm{b}}(\mathbf{k})+q \mathbf{A}\right)^{2}-q \mathbf{S} \cdot\left[a(i \mathbf{k} \times \mathbf{f})+a(i \mathbf{k} \times \mathbf{f})^{*}\right] \\
& \geq\left(2 C_{1} \Lambda-2|q| \epsilon \Lambda-4 q^{2} G(\mathbf{f})-\left(2+\epsilon^{\prime}+\frac{1}{\epsilon^{\prime}}\right)\left\langle\omega^{-1 / 2} \mathbf{f}, \omega^{-1 / 2} \mathbf{f}\right\rangle\right) \mathrm{d} \Gamma_{\mathrm{b}}(\omega) \\
& \quad+C_{1}^{2} \Lambda^{2}-\frac{|q|}{2 \epsilon \Lambda}\langle\mathbf{f}, \omega \mathbf{f}\rangle-\left(1+\frac{1}{\epsilon^{\prime}}\right) q^{2}\langle\mathbf{f}, \mathbf{f}\rangle, \quad \epsilon, \epsilon^{\prime}>0 .
\end{aligned}
$$

It is easy to see that $\left\langle\omega^{-1 / 2} \mathbf{f}, \omega^{-1 / 2} \mathbf{f}\right\rangle=8 \pi \Lambda,\langle\mathbf{f}, \omega \mathbf{f}\rangle=8 \pi \Lambda^{3} / 3,\langle\mathbf{f}, \mathbf{f}\rangle=4 \pi \Lambda^{2}$. Hence, by the definition of $C_{1}$, the left hand side of (24) is positive for suitable $\epsilon, \epsilon^{\prime}>0$. Thus, using Lemma 2.1 (and (16)), we have

$$
H(\mathbf{p}) \geq-\sqrt{|\mathbf{p}|^{2}+M^{2}}-C_{1} \Lambda .
$$

For normalized vectors $u \in \mathbb{C}^{4}, \psi \in \operatorname{Dom}\left(\mathrm{d} \Gamma_{\mathrm{b}}(\omega)\right)$ we define

$$
\begin{aligned}
& a_{j}:=\left\langle u, \alpha_{j} u\right\rangle, \quad h(\mathbf{a}):=\mathrm{d} \Gamma_{\mathrm{b}}(\omega-\mathbf{a} \cdot \mathbf{k})-q \mathbf{a} \cdot \mathbf{A}, \\
& \Psi:=u \otimes \psi \in \mathcal{F} .
\end{aligned}
$$

Note that $\omega-\mathbf{a} \cdot \mathbf{k} \geq 0$ and $\omega-\mathbf{a} \cdot \mathbf{k}$ is injective as a multiplication operator. We have

$$
\langle\Psi, H(\mathbf{p}) \Psi\rangle=\mathbf{a} \cdot \mathbf{p}+M\langle u, \beta u\rangle+\langle\psi, h(\mathbf{a}) \psi\rangle
$$

Since $h(\mathbf{a})$ is a van Hove type Hamiltonian, we have

$$
\begin{aligned}
\inf \sigma(h(\mathbf{a})) & =-q^{2}\left\|(|\mathbf{k}|-\mathbf{a} \cdot \mathbf{k})^{-1 / 2} \mathbf{a} \cdot \mathbf{f}\right\|^{2} \\
& =-4 \pi \Lambda q^{2}+q^{2}\left(1-|\mathbf{a}|^{2}\right) \int_{\mathbb{R}^{3}} \mathrm{~d} \mathbf{k} \frac{\chi_{\Lambda}(\mathbf{k})}{|\mathbf{k}|^{2}-(\mathbf{a} \cdot \mathbf{k})^{2}} \\
& =-4 \pi \Lambda q^{2}+2 \pi \Lambda q^{2}\left(1-|\mathbf{a}|^{2}\right) \frac{1}{|\mathbf{a}|} \log \left(\frac{1-|\mathbf{a}|}{1+|\mathbf{a}|}\right),
\end{aligned}
$$

where $\sigma$ means the spectrum(e.g. [3]). Thus we have

$$
E_{0}(\mathbf{p}) \leq \inf _{u \in \mathbb{C}^{4} ;\|u\|=1} \inf _{\psi \in \operatorname{Dom}\left(\mathrm{d} \Gamma_{\mathrm{b}}(\omega)\right)}\langle\Psi, H(\mathbf{p}) \Psi\rangle=\inf _{u \in \mathbb{C}^{4} ;\|u\|=1} \mathcal{E}(u)
$$

\section{Acknowledgements}

The author is grateful to Professor A. Arai of Hokkaido university for helpful comments. 


\section{References}

[1] A. Arai, Fundamental Properties of the Hamiltonian of a Dirac Particle Coupled to the Quantized Radiation Field, Hokkaido Univ. Preprint Series in Math, No. 447, 1999.

[2] A. Arai, A particle-field Hamiltonian in relativistic quantum electrodynamics, $J$. Math. Phys. 41 (2000), 4271-4283.

[3] A. Arai, Fock Spaces and Quantum Fields, Nippon-Hyouronsha, Tokyo, 2000(in Japanese).

[4] M. Reed and B. Simon, Methods of Modern Mathematical Physics Vol. II, Academic Press, New York, 1975. 